\title{
Method of Left Lymph Node Dissection
}

National Cancer Institute

\section{Source}

National Cancer Institute. Method of Left Lymph Node Dissection. NCI Thesaurus. Code C159264.

A request to identify the method employed to dissect the left lymph node. 